\title{
Unilateral hypoglossal nerve and recurrent laryngeal nerve weakness following endotracheal intubation
}

\begin{abstract}
Endotracheal intubation with cuffed tube is a safe procedure associated with few complications in majority of patients. Immediate complications are primarily associated with problems dring intubation and extubation while early and late complications represent the short- and long-term effets of epithelial trauma. True vocal cord paralysis may follow endotracheal intubation and be the result of peripheral nerve damage. This damage can occur as the result of compressing the nerve between an inflated endotracheal tube cuff and the overlying thyroid cartilage. ${ }^{1}$

The hypoglossal nerve ( $12^{\text {th }}$ cranial nerve) is motor nerve. It supplys the tongue muscle and help in speech, Food manipulation, and swallowing. Hypoglossal nerve injury following endotracheal intubation under general anesthesia is a rare complication and can cause symptoms, such as dysarthria and dysphagia. ${ }^{2}$
\end{abstract}

Keywords: Endotracheal intubation, recurrent laryngeal nerve, hypoglossal nerve, palsy, paralysis, complications
Volume II Issue 3 - 2019

\author{
Walaa Ali Kheir \\ Emergency Medicine, Qatar
}

Correspondence: Walaa Ali Kheir, Emergency Medicine, Qatar, Email wilzkhei@gmail.com

Received: December 08, 2019 | Published: December 12, 2019

\section{Abbreviations: ETT, Endotracheal Tube}

\section{Case presentation}

A 30 years old female not known to have any medical illness was admitted electively for septoplasty. Procedure done under general anesthesia, patient was easily intubated with induction of propofol, fentanyl and paralytic agent. Using size 7 ETT cuffed tube with size 3 blade, there were no difficulties in the laryngoscopic view, tube was fixed at the level of $23 \mathrm{~cm}$ at incisors. Procedure was un eventful and the patient then shifted to the post-operative ward. Two days post-operative, patient developed slurred speech and difficulty of swallowing with hoarseness of voice, examination showed the tongue was curved toward the left side consistent with left hypoglossal nerve palsy. The patient was seen by neurology who ordered MRI head / MRA head and neck which came to be normal. ${ }^{3,4}$

\section{In hospital course:}

Patient followed up regularly in the clinic by speech therapist, the hypoglossal nerve palsy improved totally but vocal cord paly still present. The case was sent abroad for an overseas specialized center in voice rehabilitation for more management.

\section{Treatment and outcome:}

Patient underwent augmentation of the left vocal cord and speech therapy, she had partially improved, but she is still has hoarseness of voice. Still following with speech therapy.

Endoscopic vocal cord examination pictures (one year after the injury) as shown down:

Lt vocal cord weakness with thickening post augmentation. Figures

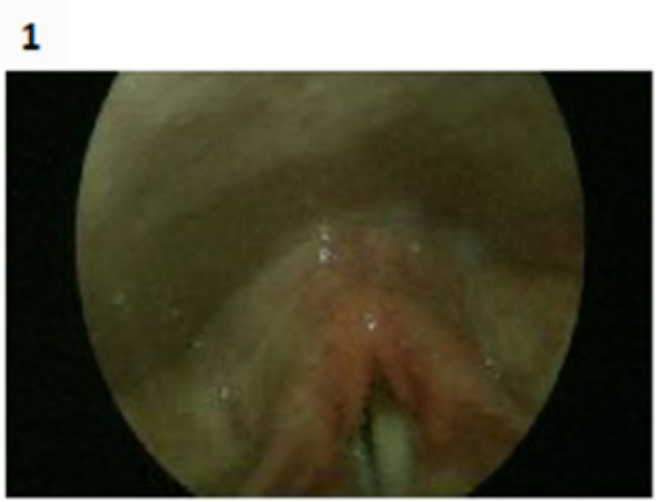

2

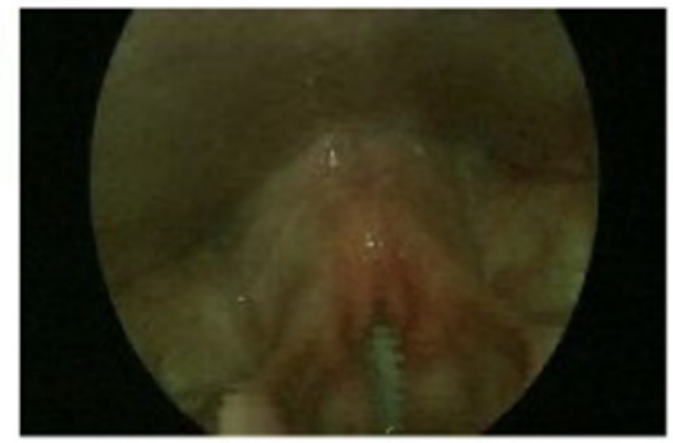




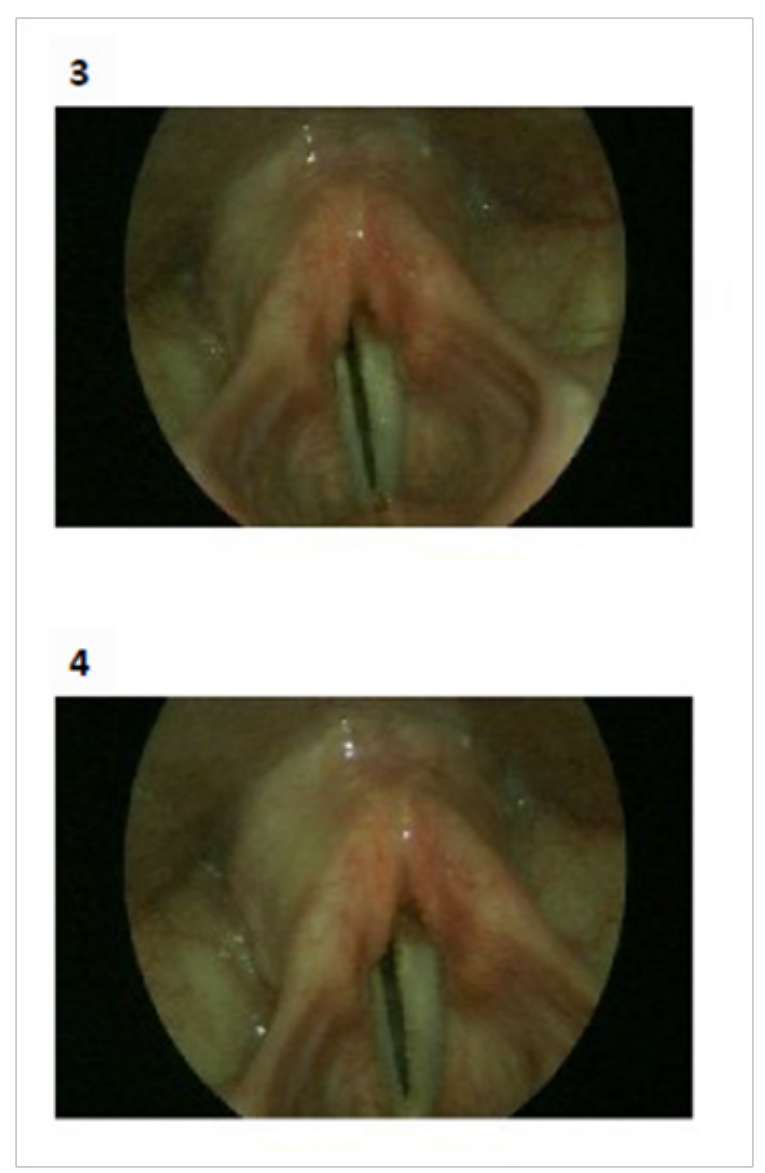

\section{Discussion and conclusion}

In our case, complete recovery of function from hypoglossal nerve palsy is generally achieved within the first 2 weeks.

In conclusion, the cause of hypoglossal nerve palsy in our patient was assumed to be a complication of direct compression of the hypoglossal nerve beneath the angle of the mandible during the surgical procedure with mask ventilation and oropharyngeal manipulation. Therefore, in order to prevent this complication, we must apply gentl direct laryngoscopy and carefull manipulation the patient's head and neck. True vocal cord paralysis may follow endotracheal intubation and be the result of peripheral nerve damage. This damage can occur as the result of compressing the nerve between an inflated endotracheal tube cuff and the overlying thyroid cartilage.

\section{Conflicts of interest}

I have no conflict of interest in relation to this case.

\section{References}

1. Ellis PD, Pallister WK. Recurrent laryngeal nerve palsy and endotracheal intubation. J Laryngol Otol. 1975;89(8):823-826.

2. Hong SJ, Lee JY. Isolated unilateral paralysis of the hypoglossal nerve after transoral intubation for general anesthesia. Dysphagia. 2009;24(3):354-356.

3. Hung NK, Lee $\mathrm{CH}$, Chan SM, et al. Transient unilateral hypoglossal nerve palsy after orotracheal intubation for general anesthesia. Acta Anaesthesiol Taiwan. 2009;47(1):48-50.

4. Cavo Jr JW. True vocal cord paralysis following intubation. Laryngoscope. 1985;95(11):1352-1359. 